\title{
Low dose dobutamine echocardiography for predicting functional recovery after coronary revascularisation
}

F Piscione, P Perrone-Filardi, G De Luca, M Prastaro, C Indolfi, P Golino, $S$ Dellegrottaglie, M Chiariello

\begin{abstract}
Objective-To evaluate the effects of chronic coronary occlusion on the accuracy of low dose dobutamine echocardiography in predicting recovery of dysfunctional myocardium after revascularisation.

Design-Retrospective study.

Setting-Tertiary referral centre.

Patients-53 consecutive patients with $\geqslant 70 \%$ stenosis of the left anterior descending coronary artery (LAD) and regional ventricular dysfunction (group 1, non-occluded LAD; group 2, occluded LAD) who underwent dobutamine echocardiography.

Interventions-26 patients underwent coronary artery bypass grafting and 27 had percutaneous transluminal coronary angioplasty.

Main outcome measures-Baseline studies before revascularisation included cross sectional echocardiography at rest and during dobutamine infusion $(5-10 \mu \mathrm{g} / \mathrm{kg} / \mathrm{min})$, and coronary angiography. The dobutamine study was performed mean (SD) 35 (28) days before revascularisation. Echocardiography at rest was repeated 90 (48) days after revascularisation.

Results-Of 296 dysfunctional segments, 63 in group 1 (43\%; 63/146) and 69 in group 2 (46\%; 69/150) (NS) improved at follow up. Mean (SD) regional wall motion score index decreased from 1.97 (0.48) (95\% confidence interval (CI) 1.01 to 2.93 ) before revascularisation to 1.74 (0.52) (95\% CI 0.70 to 2.78) at follow up in group $1(\mathrm{p}=0.001)$, and from $2.12(0.41)(95 \% \mathrm{CI}$ 1.30 to 2.98$)$ to $1.88(0.36)$ (95\% CI 1.16 to 2.60$)$ in group $2(\mathrm{p}=0.0006)$. In group 1 , sensitivity $(87 \%$ v $52 \% ; \mathrm{p}<0.0001)$, negative predictive value $(88 \% v 65 \% ; \mathrm{p}=0.001)$, and accuracy $(77 \% v 64 \% ; \mathrm{p}=0.01)$ were all significantly higher than in group 2 , despite the angiographic evidence of collaterals in patients with occluded vessels.

Conclusions-Dobutamine echocardiography shows reduced sensitivity in predicting recovery of dysfunctional myocardium supplied by totally occluded vessels. Thus caution should be used in selecting such patients for revascularisation on the basis of a viability assessment made in this way. (Heart 2001;86:679-686)
\end{abstract}

Keywords: dobutamine; coronary artery disease; viability; chronic occlusion

Dobutamine echocardiography is currently used to predict recovery of regional and global left ventricular systolic function in patients with chronic coronary artery disease. Inotropic stimulation with dobutamine results in a transient amelioration of contractile dysfunction in viable myocardium which is highly predictive of functional recovery following revascularisation. ${ }^{1-6}$ However, although the specificity of the test is very high its sensitivity has been reported to be suboptimal, especially in the setting of severely dysfunctional myocardium. ${ }^{4}$ It has been shown that enhancement of contractile function by dobutamine relies on preservation of coronary flow reserve, leading to an increase in blood flow in dysfunctional myocardium. ${ }^{89}$ However, coronary reserve is correlated with the severity of coronary artery stenosis and is greatly reduced in territories supplied by occluded vessels. ${ }^{11}{ }^{11}$ Thus it is conceivable that the accuracy of the test may vary in relation to the patency of the vessel, but very limited data evaluating such a hypothesis have so far been reported. ${ }^{12}$

The aim of our present study was to evaluate whether the accuracy of low dose dobutamine echocardiography is influenced by the patency of coronary vessels and by the development of a collateral circulation in patients with chronic coronary artery disease. We selected patients with left anterior descending coronary artery (LAD) disease because revascularisation of the LAD has a greater impact on left ventricular function and prognosis, ${ }^{13-15}$ and because it minimises the source of overlap among different vascular territories.

\section{Methods}

PATIENT POPULATION

Our patient population was selected from a consecutive group of 170 patients who formed part of a prospective protocol at our institution. The criteria for entering the study were first, the presence of $\geqslant 70 \%$ diameter stenosis in the LAD; and second, regional wall motion abnormalities at rest in one or more myocardial segments belonging to the LAD territory, identified on both cross sectional echocardiography and contrast ventriculography.

Patients with myocardial infarction, unstable angina, or congestive heart failure occurring within one month of the study were excluded; additional exclusion criteria were: $\geqslant 50 \%$ left main stenosis, significant valvar disease, 
previous coronary artery bypass graft surgery, and technically inadequate echocardiographic images.

One hundred and twenty nine patients (120 men and nine women) met the entry criteria, and 110 of these $(85 \%)$ had a history of previous myocardial infarction. In 90 of the latter $(70 \%)$, the infarct occurred in the LAD territory. Among the 129 patients, an occluded LAD was present in 51 and a significantly stenosed LAD in the remaining 78. Sixty patients belonging to this initial population were revascularised. The decision to carry out revascularisation was based on stable angina in 35 patients (58\%), angina at rest in nine $(15 \%)$, a positive stress test in $11(18 \%)$, and worsening heart failure in the remaining five $(8 \%)$, but not on myocardial viability. Seven patients refused to have echocardiographic studies after revascularisation. Therefore the remaining 53 patients (50 men and three women, mean (SD) age 59 (7) years) represent the study population. This was divided in group 1 (non-occluded LAD; 27 patients; mean stenosis $82(12) \%$ ) and group 2 (occluded LAD; 26 patients). As detailed in table 1 , the two groups did not differ with respect to age, history of anterior infarction, clinical symptoms, prevalence of multivessel disease, baseline left ventricular ejection fraction, or preoperative global and regional (LAD territory) wall motion score index (WMSI).

\section{STUDY PROTOCOL}

Baseline studies before revascularisation included cross sectional echocardiography at rest and during dobutamine infusion. Forty six patients also underwent ${ }^{201}$ thallium SPECT (single photon emission computed tomography) according to the rest-redistribution protocol $^{4}$ and had coronary angiography. ${ }^{201}$ Thallium SPECT and dobutamine echocardiography were performed on the same day in most patients $(95 \%)$. They were performed at a mean of 35 (28) days before revascularisation. Echocardiography at rest was repeated 90 (48) days after revascularisation. In all patients, calcium antagonists and oral nitrates were withdrawn for at least 48 hours, $\beta$ blockers for at least 72 hours,

Table 1 Characteristics of the patient population

\begin{tabular}{llll}
\hline & $\begin{array}{l}\text { Non-occluded LAD } \\
\text { (group 1, } n=27)\end{array}$ & $\begin{array}{l}\text { Occluded LAD } \\
\text { (group 2, } n=26)\end{array}$ & $p$ Value \\
\hline $\begin{array}{llll}\text { Clinical data } \\
\text { Age (years) }\end{array}$ & $59(6)$ & $58(7)$ & NS \\
Male & 26 & 24 & NS \\
$\begin{array}{l}\text { Female } \\
\text { Hypertension }\end{array}$ & $19(31 \%)$ & 2 & NS \\
$\begin{array}{l}\text { Hypercholesterolaemia } \\
\text { Previous anterior MI }\end{array}$ & $11(40 \%)$ & $10(37 \%)$ & NS \\
$\quad \begin{array}{l}\text { Q wave } \\
\quad \text { Non-Q-wave }\end{array}$ & $16(59 \%)$ & $16(61 \%)$ & NS \\
$\begin{array}{l}\text { Angiographic data } \\
\text { Multivessel disease }\end{array}$ & $4(15 \%)$ & $3(11 \%)$ & NS \\
$\begin{array}{l}\text { Proximal stenosis } \\
\text { Diameter stenosis (\%) }\end{array}$ & $21(77 \%)$ & $20(78 \%)$ & NS \\
$\begin{array}{l}\text { Collateral channels to LAD } \\
\text { Echocardiographic data }\end{array}$ & $17(63 \%)$ & $16(62 \%)$ & NS \\
LVEF (\%) & $1(3 \%)$ & 100 & $\mathrm{p}<0.001$ \\
WMSI & $44(11)$ & $22(85 \%)$ & $\mathrm{p}<0.001$ \\
Regional WMSI & $1.68(0.33)$ & $41(9)$ & $\mathrm{NS}$ \\
\hline
\end{tabular}

Values are mean (SD) or $\mathrm{n}(\%)$.

LAD, left anterior descending coronary artery; LVEF, left ventricular ejection fraction; MI, myocardial infarction; WMSI, wall motion score index. and transdermal nitrates for at least 12 hours before the study. Informed consent was obtained from each patient before the study.

\section{ECHOCARDIOGRAPHY}

Cross sectional echocardiography was performed with a $2.5 \mathrm{MHz}$ transducer and a commercially available scanner (Sonos 1000, Hewlett-Packard Inc, Andover, Massachusetts, USA) under resting conditions and in the last three minutes of each dobutamine infusion level. Echocardiographic images were acquired in the left lateral decubitus position and recorded on $12.5 \mathrm{~mm}$ VHS videotape. Four standard views of the left ventricle were obtained for each acquisition: parasternal long axis, short axis at mitral and papillary muscle level, and apical four and two chamber views.

After baseline echocardiography had been performed, low dose dobutamine echocardiography was begun, using a mechanical infusion pump. The initial dose was $5 \mu \mathrm{g} / \mathrm{kg} / \mathrm{min}$ for five minutes, and the dose was then increased to $10 \mu \mathrm{g} / \mathrm{kg} / \mathrm{min}$ for five more minutes. Blood pressure was measured periodically, and a 12 lead ECG was monitored continuously throughout the study and during the recovery phase.

The total duration of the study did not exceed 30 minutes. No patient reported angina or developed life threatening arrhythmias or hypertension during the test. In two patients, dobutamine infusion had to be interrupted in the last minute of the peak dose infusion because of the development of ventricular bigeminy; this subsided after the infusion of $\beta$ blockers. Mean (SD) heart rate was 76 (10) beats/min at baseline ( $95 \%$ confidence interval (CI) 55 to 97) and increased to 95 (20) beats/ min (95\% CI 55 to 135$)$ at the end of peak infusion $(p<0.0005)$. Systolic blood pressure was 131 (20) $\mathrm{mm} \mathrm{Hg}(95 \%$ CI 91 to 171$)$ at baseline and 136 (20) $\mathrm{mm} \mathrm{Hg}$ (95\% CI 96 to 176) at the end of the study (NS). Resting echocardiography was repeated at follow up using the same examination criteria.

${ }^{201}$ THALLIUM IMAGING

After an overnight fast, patients underwent quantitative SPECT after the administration of $3 \mathrm{mCi}$ of ${ }^{201}$ thallium intravenously under resting conditions. SPECT images were acquired 15-20 minutes and four hours after the ${ }^{201}$ thallium injection, as previously described. ${ }^{4}$ Thirty two images were acquired from a $30^{\circ}$ right anterior oblique to a $60^{\circ}$ left posterior oblique view in $6^{\circ}$ increments.

CORONARY ANGIOGRAPHY

All patients underwent cardiac catheterisation. Left ventriculography and coronary angiography were carried out by the Judkins technique. The left and right coronary arteries were imaged in multiple views, including craniocaudal angulations. To minimise the potential effect of coronary vasoconstriction, contrast injection was performed after intracoronary administration of glyceryl trinitrate $(200 \mu \mathrm{g})$. The number of diseased vessels did not differ significantly between the two groups of patients (table 1). 

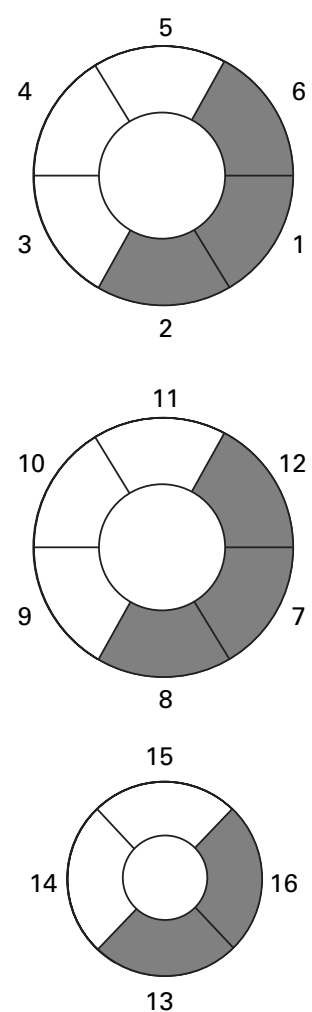

Figure 1 Pie chart showing the division of left ventricle into 16 segments in short axis tomograms at chordal (upper), mid-papillary (medium), and apical (lower) levels. White segments were assigned to the left anterior descending coronary artery ( $L A D)$ territory.

ECHOCARDIOGRAPHIC ANALYSIS

Undigitised echocardiographic images were analysed off line from the videotape playback by continuous display. This was done by two independent operators who were unaware of the clinical and angiographic results. When echocardiography was performed during follow up, the operators were not aware of the pre-revascularisation study results.

Regional wall motion was evaluated at the chordal, papillary muscle, and apical levels, and was assessed visually using both endocardial motion and wall thickening. For analysis, the left ventricle was divided into 16 segments as recommended by the American Society of Echocardiography. ${ }^{16}$ The short axis midventricular images at the cordal and midpapillary muscle levels were divided into six myocardial segments, starting at the 3 o'clock position and proceeding clockwise, representing posterolateral, inferior, posteroseptal, anteroseptal, anterior, and anterolateral myocardium, respectively. Short axis images at the apical level were divided into four myocardial segments, starting at the half past one o'clock position and proceeding clockwise, representing lateral, inferior, septal, and anterior apical myocardium. The LAD territory was defined as basal, mid, and distal segments of the left anterior wall, basal and mid segments of the anterior and posterior septum, and the distal septum, the total number of segments being eight (fig 1). To evaluate regional systolic function, wall motion and thickening were graded semiquantitatively using a scoring system where 1 = normal contraction, 2 = hypokinesia (severely reduced wall thickening and inward motion), 3 = akinesia (absence of wall motion and of systolic thickening), and $4=$ dyskinesia (absence of wall thickening and outward motion). Systolic function was scored at rest and at each dobutamine dose.

Regional systolic dysfunction was defined when a score of $\geqslant 2$ was assigned to a myocardial segment in at least two different echocardiographic views. Viable myocardium was defined as an improvement of functional score of $\geqslant 1$ grade in at least one segment with rest hypokinesia or akinesia. Myocardial scarring was identified in segments with resting dysfunction that did not show improvement during low dose dobutamine echocardiography. Recovery in the LAD territory was defined as improvement in functional score by $\geqslant 1$ grade of one or more septal or anterior segments compared with the preoperative rest study. For both preoperative and postoperative rest echocardiograms, a WMSI was derived (the sum of individual wall motion scores divided by the respective number of segments) for the entire ventricle (global WMSI) and for the LAD territory (regional WMSI).

The reproducibility of the echocardiographic analysis in our laboratory has been reported previously. ${ }^{4}$ In particular, exact agreement in segmental scores (including function at rest and during inotropic stimulation) was $82 \%$ ( $\kappa$ value $0.62 ; \mathrm{p}<0.01$ ), and in $97.5 \%$ of all segments analysed the score difference between two echocardiographic readings was within 1 point.

Our interobserver and intraobserver reproducibility of the global left ventricular ejection fraction was analysed by the Bland-Altman method. This analysis showed no significant difference for either interobserver variability $(r=0.37 ; \mathrm{NS})$ or intraobserver variability $(r=0.3 ; \mathrm{NS})$. According to our variability data, a cut off point of $6 \%$ was chosen to distinguish patients with or without a significant improvement in postrevascularisation ejection fraction.

\section{${ }^{201}$ THALLIUM IMAGING}

In each patient, four consecutive midventricular slices from the short axis series were considered. Tomograms were then divided into six sectors of equal arc, representing the posterolateral, inferior, posteroseptal, anteroseptal, anterior, and anterolateral myocardium. ${ }^{4}$ The sectors from two consecutive three pixel thick short axis tomograms were then grouped and averaged. Thus 12 myocardial regions were analysed for each patient. Of these segments, six were assigned to the territory of the LAD, four (two anterolateral and two posterolateral) were assigned to the territory of left circumflex coronary artery, and two inferior segments were assigned to the territory of the right coronary artery (fig 1). Alignment and analysis of the studies were visually made by two operators unaware of the echocardiographic results.

A cut off point of $65 \%$ was used to distinguish viable from non-viable myocardium. ${ }^{17}$ 


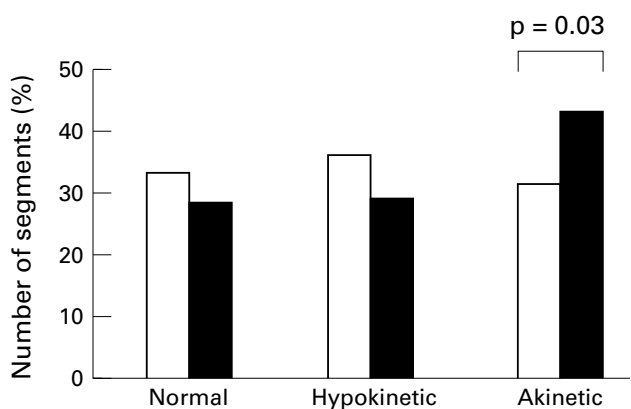

Figure 2 Regional systolic function represented as the percentage of segments showing normal contraction, hypokinesia, and akinesia in patients with a non-occluded left anterior descending coronary artery ( $L A D$; white bars) and an occluded LAD (black bars). The percentage of akinetic segments was significantly higher in patients with an occluded LAD.

CORONARY ANGIOGRAPHY ANALYSIS

All coronary angiograms were analysed by an investigator who had no knowledge of the results of the echocardiograms. Each vessel was visualised in multiple views, and the most severe appearing lesion was selected for analysis. Care was taken to avoid overlap of adjacent branches. Vessel occlusion was defined as a lesion with no anterograde distal flow. Collateral circulation was graded according to the classification of Rentrop and colleagues ${ }^{18}: 0=$ no visible collateral, $1=$ collateral filling of the vessel branches without reaching the epicardial artery, 2 = partial filling of the epicardial artery, and $3=$ complete filling of the epicardial artery.

STATISTICAL ANALYSIS

Statistical analysis was performed with the SPSS statistical package. ${ }^{19}$ Unpaired and paired $t$ tests were used to compare continuous variables. Values are reported as mean (SD) and $95 \%$ confidence intervals. Bonferroni's correction was used for multiple group comparisons.

\section{Results}

Angiographic collateral flow to the LAD was present in one patient in group 1 without occlusion $(1 / 27 ; 3 \%)$ and in 22 patients in group $2(22 / 26 ; 85 \%)$ with an occluded LAD $(p<0.00001)$. There were no significant differences in clinical characteristics between the two groups (table 1). The left ventricular ejection fraction was $44(11) \%(95 \%$ CI $22 \%$ to $66 \%)$ in group 1 , and 41 (9)\% (95\% CI $23 \%$ to $59 \%$ ) in group 2 (NS).

Twenty seven patients underwent transluminal coronary angioplasty (PTCA; 11 patients in group 1 and 16 patients in group 2); successful dilatation - defined as a diameter of the residual stenosis not exceeding $30 \%$ of the lumen diameter-was achieved in all cases. The remaining 26 patients underwent coronary artery bypass grafting (CABG; 16 in group 1 and 10 in group 2); in all these cases grafting of the LAD was successful. The internal mammary artery was used in 10 patients (six in group 1 and four in group 2). No patients had major complications associated with the revascularisation procedure.

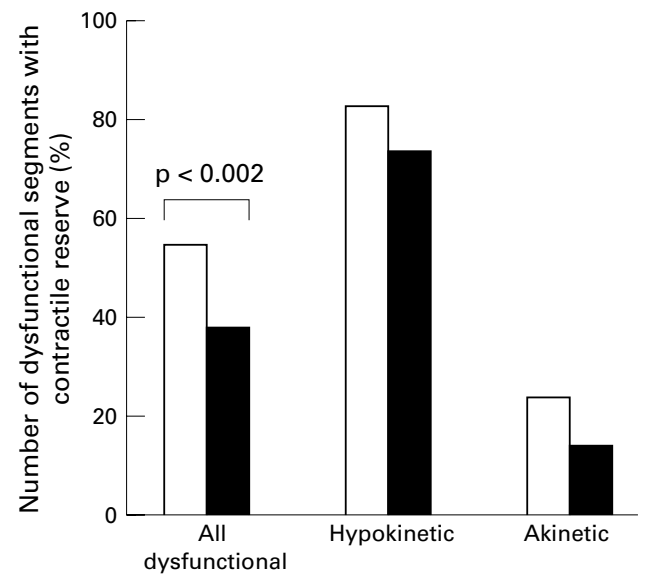

Figure 3 Percentage of dysfunctional segments showing contractile reserve on low dose dobutamine echocardiography in patients with a non-occluded left anterior descending coronary artery ( $L A D$; white bars) and an occluded LAD (black bars).

In all, we evaluated 424 myocardial segments belonging to the LAD territory; $296(70 \%)$ of these were dysfunctional, including 146 in group 1 and 150 in group 2 . Figure 2 shows the data on systolic function in the segments evaluated.

\section{SEGMENTAL ANALYSIS}

In group 1 patients, 79 (54\%) of 146 dysfunctional segments were hypokinetic and 67 $(46 \%)$ were akinetic. In group 2, $60(40 \%)$ of 150 dysfunctional segments were hypokinetic (NS $v$ group 1) and $90(60 \%)$ were akinetic $(\mathrm{p}=0.03 v$ group 1$)$.

The number of dysfunctional segments showing contractile reserve during low dose dobutamine echocardiography was significantly higher in group 1 than in group 2 patients (81 of $146(55 \%) v 57$ of $150(38 \%)$; $\mathrm{p}=0.002$; fig 3 ). This difference was mostly accounted for by the higher percentage of akinetic segments showing contractile reserve in group 1 patients than in group 2 patients, although this difference was not significant $(16 / 67(24 \%) v 13 / 90$ (14\%), respectively) (fig $3)$.

A biphasic response was observed in only three segments $(1 \%)$, one in group 1 and two in group 2).

Of 296 dysfunctional segments in the LAD territory undergoing revascularisation, 132 (44\%) improved at follow up. Recovery of regional function was not affected by the patency of the coronary vessel, as it occurred in $63(43 \%)$ of 146 dysfunctional segments in group 1 and $69(46 \%)$ of 150 dysfunctional segments in group 2 (NS). In particular, improvement was observed in $45(57 \%)$ of 79 hypokinetic segments in group 1 and in 35 (58\%) of 60 hypokinetic segments in group 2 (NS), as well as in $18(27 \%)$ of 67 akinetic segments in group 1 and 34 (38\%) of 90 akinetic segments in group 2 (NS) (fig 4).

Sensitivity, specificity, accuracy, and positive and negative predictive power for dobutamine echocardiography and ${ }^{201}$ thallium SPECT in predicting functional recovery after revascularisation are reported in table 2. Low dose 


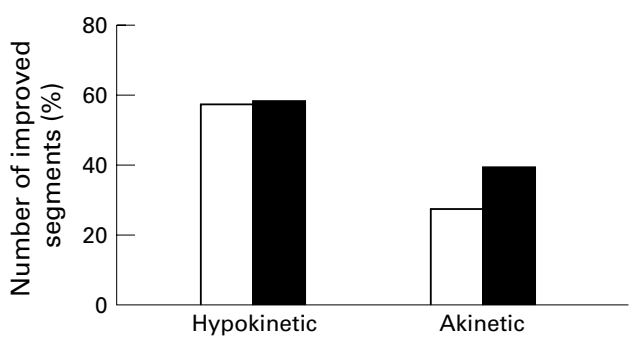

Figure 4 Effects of revascularisation on regional systolic function. The percentage of improved hypokinetic and akinetic segments did not differ between patients with a non-occluded left anterior descending coronary artery (LAD; white bars) and an occluded LAD (black bars).

dobutamine echocardiography showed a better sensitivity ( $87 \%$ v 52\%; $\mathrm{p}<0.0001)$, accuracy $(77 \%$ v $64 \% ; \mathrm{p}=0.01)$, and negative predictive value $(88 \% v 65 \% ; \mathrm{p}=0.001)$ in group 1 than in group 2, respectively. These data were confirmed when further analysis was restricted to akinetic segments (table 3).

A further analysis was done by dividing territories with or without $\mathrm{Q}$ wave myocardial infarction in the two groups; this did not show any significant differences (table 4 ).

Table 2 Accuracy of low dose dobutamine echocardiography (LDDE) and ${ }^{201}$ thallium SPECT in predicting functional recovery after revascularisation in patients with a non-occluded (group 1) and an occluded (group 2) left anterior descending coronary artery

\begin{tabular}{|c|c|c|c|c|}
\hline & \multicolumn{2}{|l|}{$L D D E$} & \multicolumn{2}{|c|}{${ }^{201}$ Thallium SPECT } \\
\hline & Group 1 & Group 2 & Group 1 & Group 2 \\
\hline Sensitivity & $87 \%(55 / 63)$ & $52 \%(36 / 69)^{\star \star \star \star}$ & $80 \%(48 / 60)$ & $72 \%(39 / 54) \dagger$ \\
\hline Specificity & $68 \%(57 / 83)$ & $74 \%(60 / 81)$ & $21 \%(7 / 33)+\dagger \dagger$ & $64 \%(23 / 36)^{\star \star \star}$ \\
\hline Accuracy & $77 \%(112 / 146)$ & $64 \%(96 / 150)^{\star}$ & $59 \%(55 / 93)+t$ & $69 \%(62 / 90)$ \\
\hline PPV & $68 \%(55 / 81)$ & $63 \%(36 / 57)$ & $65 \%(48 / 74)$ & $75 \%(39 / 52)$ \\
\hline NPV & $88 \%(57 / 65)$ & $65 \%(60 / 93)^{\star \star}$ & $37 \%(7 / 19)+\dagger+$ & $61 \%(23 / 38)$ \\
\hline
\end{tabular}

${ }^{\star} \mathrm{p}=0.01 ;{ }^{\star \star} \mathrm{p}=0.001 ;{ }^{\star \star \star} \mathrm{p}<0.0001 v$ group 1 .

tp $<0.05 ;$ ttp $<0.01 ;$ tttp $<0.0001 v$ LDDE.

$\mathrm{NPV}$, negative predictive value; PPV, positive predictive value; SPECT, single photon emission computed tomography.

Table 3 Accuracy of low dose dobutamine echocardiography (LDDE) and ${ }^{201}$ thallium SPECT in predicting functional recovery after revascularisation of akinetic segments in patients with a non-occluded (group 1) and an occluded (group 2) left anterior descending coronary artery

\begin{tabular}{llllll}
\hline & \multirow{2}{l}{ LDDE } & & & \multicolumn{2}{l}{${ }^{201}$ Thallium SPECT } \\
\cline { 2 - 3 } \cline { 5 - 6 } \cline { 5 - 6 } & Group 1 & Group 2 & & Group 1 & Group 2 \\
\hline Sensitivity & $72 \%(13 / 18)$ & $26 \%(9 / 34)^{\star \star \star}$ & & $67 \%(10 / 15)$ & $79 \%(19 / 24)+\dagger \dagger$ \\
Specificity & $94 \%(46 / 49)$ & $93 \%(52 / 56)$ & & $26 \%(5 / 19) \dagger+\dagger$ & $60 \%(9 / 15)+\dagger$ \\
Accuracy & $88 \%(59 / 67)$ & $68 \%(61 / 90)^{\star \star}$ & & $44 \%(15 / 34) \dagger$ & $72 \%(28 / 39)^{\star}$ \\
PPV & $81 \%(13 / 16)$ & $69 \%(9 / 13)$ & & $42 \%(10 / 24) \dagger$ & $76 \%(19 / 25)^{\star}$ \\
NPV & $90 \%(46 / 51)$ & $67 \%(52 / 77)^{\star \star}$ & & $50 \%(5 / 10) \dagger$ & $64 \%(9 / 14)$ \\
\hline
\end{tabular}

${ }^{\star} \mathrm{p}<0.05 ;{ }^{\star \star} \mathrm{p}<0.01 ;{ }^{\star \star \star} \mathrm{p}<0.001 v$ group 1

tp $>0.05 ;$ t tp $<0.01 ;$ tttp $<0.001 v$ LDDE

$\mathrm{NPV}$, negative predictive value; PPV, positive predictive value; SPECT, single photon emission computed tomography.

Table 4 Accuracy of low dose dobutamine echocardiography in predicting functional recovery after revascularisation in the absence or presence of complete vascular occlusion (groups 1 and 2) and in the absence (control) or presence of a $Q$ wave anterior myocardial infarct

\begin{tabular}{|c|c|c|c|c|}
\hline & \multicolumn{2}{|l|}{ Group 1} & \multicolumn{2}{|l|}{ Group 2} \\
\hline & $Q$ wave $M I$ & Control & $Q$ wave $M I$ & Control \\
\hline Sensitivity & $89 \%(34 / 38)$ & $84 \%(21 / 25)$ & $52 \%(23 / 44)$ & $52 \%(13 / 25)$ \\
\hline Specificity & $71 \%(39 / 55)$ & $64 \%(18 / 28)$ & $74 \%(43 / 58)$ & $74 \%(17 / 23)$ \\
\hline Accuracy & $78 \%(73 / 93)$ & $73 \%(39 / 53)$ & $65 \%(66 / 102)$ & $63 \%(30 / 48)$ \\
\hline PPV & $68 \%(34 / 50)$ & $68 \%(21 / 31)$ & $61 \%(23 / 38)$ & $68 \%(13 / 19)$ \\
\hline NPV & $90 \%(39 / 43)$ & $82 \%(18 / 22)$ & $67 \%(43 / 64)$ & $59 \%(17 / 29)$ \\
\hline
\end{tabular}

Group 1, non-occluded left anterior descending coronary artery; group 2, occluded left anterior descending coronary artery; MI, myocardial infarction; NPV, negative predictive value; PPV, positive predictive value.
To investigate whether the degree of the collateral circulation influenced the dobutamine echocardiography results, patients with an occluded LAD were further subdivided into group 2A with a well developed collateral circulation ( $n=18$; Rentrop class III and II), and group 2B with a less well developed collateral circulation $(n=8$; Rentrop classes I and $0)$. In group $2 \mathrm{~A}$, the sensitivity and accuracy were significantly reduced compared with group 1 patients $(53 \%$ v $87 \%, \mathrm{p}<0.0001$; and $65 \% v 77 \%, \mathrm{p}=0.036)$, but not different from group $2 \mathrm{~B}$ ( $50 \%$ and $60 \%$, respectively). No significant differences in specificity were observed among the three groups of patients. In contrast, recovery of contractile function was not affected by the status of the coronary artery before revascularisation, as regional wall motion improved to a similar degree in the three groups of patients (table 5).

${ }^{201}$ Thallium SPECT data were available on $183(62 \%)$ of 296 dysfunctional segments (93 of group 1 and 90 of group 2). In group 2 (table 2), ${ }^{201}$ thallium SPECT showed a significantly higher sensitivity than low dose dobutamine echocardiography $(72 \%$ v 52\%; p < 0.05), whereas in the same comparison in group 1 there was lower specificity $(21 \%$ v $68 \%$; $\mathrm{p}<0.0001)$, lower accuracy (59\% v 77\%; $\mathrm{p}<0.01$ ), and reduced negative predictive value $(37 \%$ v $88 \% ; \mathrm{p}<0.0001)$. These data were also confirmed when further analysis was restricted to akinetic segments (table 3 ).

PATIENT ANALYSIS

In the whole group of patients, left ventricular ejection fraction increased significantly from 43 (9) $\%$ (95\% CI $25 \%$ to $61 \%$ ) before revascularisation to 47 (9)\% (95\% CI $29 \%$ to $65 \%)$ afterwards $(\mathrm{p}<0.001)$. Left ventricular ejection fraction improved similarly in patients undergoing PTCA (from 43 (10)\% (95\% CI $23 \%$ to $63 \%)$ to $47(10) \%(95 \%$ CI $27 \%$ to $67 \%))(\mathrm{p}<0.001)$ and in those subjected to CABG (from 41 (11)\% (95\% CI 19\% to 63\%) to $45(11) \%(95 \%$ CI $23 \%$ to $67 \%))$ $(\mathrm{p}<0.001)$.

Improvement in left ventricular ejection fraction was more pronounced in group 2 patients (from 41 (9)\% (95\% CI 23\% to $59 \%$ ) to $47 \quad(7) \% \quad(95 \%$ CI $33 \%$ to $61 \%))$ $(\mathrm{p}<0.001)$, whereas this did not reach significance in group 1 patients (from 44 (11)\% (95\% CI $22 \%$ to $66 \%)$ to $46(10) \%$ (95\% CI $26 \%$ to $66 \%$ )) (NS).

We compared the accuracy of low dose dobutamine echocardiography and ${ }^{201}$ thallium SPECT in predicting global left ventricular function after revascularisation in patients with single vessel revascularisation. These data are reported in table 6 . We found a threshold of at least two viable segments conferring optimal values of accuracy. In group 1, low dose dobutamine echocardiography showed a similar value for sensitivity but a higher value for accuracy than ${ }^{201}$ thallium SPECT, whereas in group 2, ${ }^{201}$ thallium SPECT showed better sensitivity ( $89 \%$ v 44\%) and accuracy $(60 \% v$ $57 \%$ ) than low dose dobutamine echocardiography. 
Table 5 Accuracy of low dose dobutamine echocardiography (LDDE) and the effects of revascularisation in patients with a non-occluded left anterior descending coronary artery (LAD) (group 1), an occluded LAD and collaterals of class II-III (group $2 A$ ), and an occluded LAD and collaterals of class $0-I$ (group $2 B$ )

\begin{tabular}{llll}
\hline & Group 1 & Group $2 A$ & Group $2 B$ \\
\hline LDDE sensitivity & $87 \%$ & $53 \%{ }^{\star \star}$ & $50 \%$ \\
LDDE specificity & $69 \%$ & $73 \%$ & $75 \%$ \\
LDDE accuracy & $77 \%$ & $65 \% \star$ & $60 \%$ \\
Regional WMSI & $1.97(0.48)$ & $2.26(0.33)$ & $1.81(0.52)$ \\
$\quad$ Pre- & $1.74(0.53)+\dagger$ & $2.01(0.30)+\dagger$ & $1.59(0.36) \dagger$ \\
Post- & 12 & 9 & 12 \\
Regional WMSI change (\%) & & & \\
\hline
\end{tabular}

$\star_{\mathrm{p}}<0.05 ;{ }^{\star} \mathrm{p}<0.0001 v$ group 1

$t \mathrm{p}<0.05 ;+t \mathrm{p}<0.01 v$ regional WMSI pre-revascularisation.

WMSI, wall motion score index.

Table 6 Accuracy of low dose dobutamine echocardiography (LDDE) and ${ }^{201}$ thallium SPECT in predicting global left ventricular functional recovery after single vessel revascularisation for either a non-occluded (group 1) or an occluded (group 2) left anterior descending coronary artery

\begin{tabular}{|c|c|c|c|c|}
\hline & \multicolumn{2}{|l|}{$L D D E$} & \multicolumn{2}{|c|}{${ }^{201}$ Thallium SPECT } \\
\hline & Group 1 & Group 2 & Group 1 & Group 2 \\
\hline Sensitivity & $100 \%(4 / 4)$ & $44 \%(4 / 9)$ & $100 \%(4 / 4)$ & $89 \%(8 / 9)$ \\
\hline Specificity & $28 \%(2 / 7)$ & $66 \%(4 / 6)$ & $14 \%(1 / 7)$ & $16 \%(1 / 6)$ \\
\hline Accuracy & $54 \%(6 / 11)$ & $57 \%(8 / 15)$ & $44 \%(5 / 11)$ & $60 \%(9 / 15)$ \\
\hline PPV & $44 \%(4 / 9)$ & $66 \%(4 / 6)$ & $40 \%(4 / 10)$ & $62 \%(8 / 13)$ \\
\hline NPV & $100 \%(2 / 2)$ & $44 \%(4 / 9)$ & $100 \%(1 / 1)$ & $50 \%(1 / 2)$ \\
\hline
\end{tabular}

NPV, negative predictive value; PPV, positive predictive value; SPECT, single photon emission computed tomography.

\section{Discussion}

ACCURACY OF LOW DOSE DOBUTAMINE

ECHOCARDIOGRAPHY IN PREDICTING

REVERSIBILITY OF REGIONAL DYSFUNCTION

Our main finding in the present study was that low dose dobutamine echocardiography is not very accurate in predicting recovery of dysfunctional myocardium supplied by occluded coronary arteries. In fact, only $52 \%$ of segments supplied by an occluded LAD that improved after revascularisation were identified by dobutamine echocardiography. In addition, 35\% of the segments supplied by occluded vessels that did not show contractile reserve improved after revascularisation. Sensitivity further decreased in severely dysfunctional myocardium, as only $26 \%$ of akinetic viable segments supplied by an occluded LAD were identified by dobutamine echocardiography. Low dose dobutamine echocardiography also showed a lower sensitivity in patients with occluded vessels when global left ventricular functional recovery was analysed.

Various pathophysiological mechanisms may account for this finding. It has been reported that transient amelioration of myocardial dysfunction during inotropic stimulation-the hallmark of viability using dobutamine echocardiography-depends on the recruitment of residual coronary reserve which allows an increase of blood flow in the dysfunctional myocardium. ${ }^{9}$ However, when coronary reserve is compromised, recruitment of inotropic reserve is rapidly followed by the development of ischaemia and a deterioration in function, even in the absence of completely occluded coronary vessels. ${ }^{8}$ This hypothesis is strengthened by our ${ }^{201}$ thallium SPECT data, which showed a better sensitivity and accuracy than low dose dobutamine echocardiography only in the patients with occluded vessels.
In patients with occluded coronary vessels, flow reserve has been reported to be greatly attenuated or completely lost during dypiridamole infusion. ${ }^{10}{ }^{11}$ It is conceivable that in this case the increase in blood flow in the dysfunctional myocardium may be inadequate to cause transient contractile improvement, or it may result in a very mild and transient functional enhancement undetectable by our current semiquantitative analysis of echocardiographic images.

Experimental data further indicate that the accuracy of dobutamine for identifying dysfunctional myocardium is decreased when a substantial amount of necrotic myocardium coexists in the same territory, even when blood flow is restored at reperfusion. ${ }^{20}$ In addition, reversible degradation of the cellular contractile apparatus in long standing dysfunctional myocardium may also contribute to the lack of contractile reserve, generating a false negative response. ${ }^{21}$

Thus it is conceivable that different mechanisms may contribute to reducing the accuracy of dobutamine for identifying severely dysfunctional viable myocardium.

\section{PREVIOUS STUDIES}

Despite the theoretical limitations of the test in the setting of occluded coronary arteries, there are few data on its use in such patients. In a population of 12 patients with a chronically occluded left anterior descending coronary artery and a moderately depressed ejection fraction, Afridi and colleagues reported a positive and negative predictive accuracies for dobutamine echocardiography of $100 \%$ and $86 \%$, respectively, for improvement in dysfunctional myocardium after revascularisation, though sensitivity and specificity were not reported. ${ }^{12}$ A high negative predictive accuracy is at variance with our findings in the present study, where a suboptimal value was observed, indicating that many akinetic segments defined as non-viable by dobutamine do recover after revascularisation. The short (one month) follow up evaluation in Afridi's study may partly explain this discrepancy, as it has been reported that a longer time interval may be required for dysfunctional myocardium to recover completely. ${ }^{7}$ Thus a longer period of follow up in that study might have allowed recovery of additional dysfunctional segments, so reducing the observed negative predictive value of the test.

\section{VIABILITY IN COLLATERAL DEPENDENT}

MYOCARDIUM

Another important observation of the current study is that recovery of function occurs in about half the dysfunctional segments supplied by occluded vessels, and results in a significant increase in global left ventricular ejection fraction, that is one of the main determinants of survival in patients with coronary artery disease. However, the degree of collateral flow did not predict the occurrence of functional recovery, indicating that viability - at least in terms of the possibility of recovery of contractile function at rest-is not always associated 
with the presence of a collateral circulation on angiography.

This finding supports the observation of $\mathrm{Di}$ Carli and colleagues, ${ }^{22}$ who reported that viability evaluated by positron emission tomography is detectable in only $58 \%$ of dysfunctional regions supplied by an occluded vessel where there is angiographic evidence of collaterals. It is also consistent with the data of Sabia and associates, ${ }^{23}$ who showed a lack of correlation between the degree of wall motion improvement after revascularisation and blood flow supply before revascularisation in patients with recent myocardial infarction.

Taken together, these data indicate that angiographic evaluation of collateral circulation should not be used to predict myocardial viability in patients with chronic coronary artery disease and occluded coronary arteries who are candidates for revascularisation.

\section{STUDY LIMITATIONS}

Repeat coronary angiography was not performed in the present study. Thus restenosis at the time of follow up cannot be excluded with certainty. However, no patient developed symptoms or had evidence of ischaemia on investigation, which minimises the likelihood of restenosis. In addition, the observation that most segments with unchanged function after revascularisation were in the same vascular territories as segments that showed improved function reduces this possibility still further.

Another limitation of the study is that recovery of viable dysfunctional segments may not have been complete by three months after revascularisation. However, this would tend to reduce the negative predictive value further, making our results understated.

Visual analysis of regional function may represent a limitation of the echocardiographic technique, especially in large ventricles, whereas in the present study left ventricular function and dimensions were preserved or were only moderately abnormal.

Currently, there are no reliable quantitative methods for evaluating regional function, and such methods as there are do not represent gold standard analyses at present. In addition, visual analyses from videotapes might impair reproducibility. However, data from our laboratory indicate excellent reproducibility for videotape analysis, especially for akinetic myocardium. ${ }^{4}$

In the present study only low dobutamine doses were used. However, the biphasic contractile response induced by high dobutamine doses, while being very specific, is less sensitive at predicting functional recovery than the low dose response. ${ }^{54}$ By not using high doses, we might have missed a deterioration in the function of akinetic segments that showed improvement at low dosage; in this way we may have included among the viable myocardial segments both those showing sustained improvement (for which the positive accuracy is reduced) and those with a potential biphasic response (for which the positive accuracy is very high). This would tend to maximise sensitivity while reducing specificity. However, the sensitivity of dobutamine was suboptimal in the present study, so the use of only low doses would only diminish the impact of our findings rather than exaggerate it. Moreover, our negative accuracy in akinetic segments was $65 \%$-in other words, $35 \%$ of akinetic segments that did not improve with dobutamine recovered after revascularisation. As demonstrated by Afridi and colleagues and Panza and associates, ${ }^{52}$ the vast majority of segments showing transient improvement do so at dobutamine doses of $\leqslant 10 \mu \mathrm{g} / \mathrm{kg} / \mathrm{min}$. Thus it is very unlikely that the $35 \%$ of segments classified as negative using low doses would improve at higher doses. This gives further support to our conclusion that the use of low dobutamine doses did not affect the main findings of the study.

Finally a factor that may hamper the evaluation of segmental motion is the presence of postsurgical paradoxical septal motion. We do not believe that this could have hampered our results as more than half our patients were treated by PTCA and as a combination of both inward motion and myocardial wall thickness was used in evaluating regional function.

CLINICAL IMPLICATIONS AND CONCLUSIONS Our study shows that recovery of function occurs in a sizeable number of revascularised dysfunctional segments supplied by occluded coronary arteries. Identification of such myocardial territories remains a problem, as neither the presence of collaterals nor the response to inotropic stimulation using dobutamine echocardiography is sufficiently accurate in predicting the functional effects of revascularisation under these circumstances. However, the limited number of such patients so far reported emphasises the need for further confirmation of our findings.

1 Cigarroa CG, de Filippi CR, Brickner ME, et al. Dobutamine stress echocardiography identifies hibernating myocardium and predicts recovery of left ventricular dysfunction after coronary revascularization. Circulation 1993;88:430-6.

2 Watada $\mathrm{H}$, Ito $\mathrm{H}$, Oh $\mathrm{H}$, et al. Dobutamine stress echocardiography predicts reversible dysfunction and quantitates the extent of irreversibly damaged myocardium after reperfusion of anterior myocardial infarction. $\mathcal{F} \mathrm{Am}$ Coll Cardiol 1994;24:624-30.

3 La Canna G, Alfieri O, Giubbini R, et al. Echocardiography during infusion of dobutamine for identification of reversible dysfunction in patients with chronic coronary artery disease. $\mathcal{F}$ Am Coll Cardiol 1994;23:617-26.

4 Perrone-Filardi P, Pace L, Prastaro M, et al. Dobutamine echocardiography predicts improvement of hypoperfused dysfunctional myocardium after revascularization in patients with coronary artery disease. Circulation 1995;91:2556-65.

5 Afridi I, Kleiman NS, Raizner AE, et al. Dobutamine-6hoAfridi I, Kleiman NS, Raizner AE, et al. Dobutamine echocardiography in myocardial hibernation: optimal dose and accuracy in predicting recovery of ventricular function following coronary angioplasty. Circulation 1995;91:663-70.

6 Perrone-Filardi P, Pace L, Prastaro M, et al. Assessment of myocardial viability in patients with chronic coronary artery disease. Rest-4-hour-24-hour ${ }^{201} \mathrm{Tl}$ tomography versus dobutamine echocardiography. Circulation 1996;94: 2712-19.

7 Cornel JH, Bax JJ, Elhendy A, et al. Biphasic response to dobutamine predicts improvement of global left ventricular function after surgical revascularization in patients with stable coronary artery disease. Implications of time course of recovery on diagnostic accuracy. $\mathcal{F}$ Am Coll Cardiol 1998; 31:1002-10.

8 Chen C, Li L, Prada JV, et al. Incremental doses of dobutamine induce a biphasic response in dysfunctional left ventricular regions subtending coronary stenoses. Circulation 1995;92:756-66.

9 Sun KT, Czernin J, Krivokapich J, et al. Effects of dobutamine stimulation on myocardial blood flow, glucose metabolism, and wall motion in normal and dysfunctional myocardium. Circulation 1996;94:3146-54. 
10 Uren NG, Melin JA, De Bruyne B, et al. Relation between myocardial blood flow and the severity of coronary artery .

11 Vanoverschelde JJ, Wijns W, Deprè C, et al. Mechanisms of chronic regional postischemic dysfunction in human: new insight from the study of non infarcted collateraldependent myocardium. Circulation 1993;87:1512-23.

12 Afridi I, Main M, Grayburn PA. Accuracy of dobutamine echocardiography for detection of myocardial viability in patients with an occluded left anterior descending coronary artery. F Am Coll Cardiol 1996;28:455-9.

13 Mahmarian JJ, Pratt CM, Boyce TM, et al. The variable extent of jeopardized myocardium in patients with single vessel coronary artery disease: quantification by thallium201 single photon emission computed tomography. $\mathcal{F}$ Am Coll Cardiol 1991;17:355-62.

14 Seals AA, Pratt CM, Mahmarian JJ, et al. Relation of left ventricular dilation during acute myocardial infarction to systolic performance, diastolic dysfunction, infarct size and location. Am 7 Cardiol 1988;61:224-9.

15 Gruppo Italiano per lo Studio della Streptochinasi nell' Infarto Miocardico (GISSI). Effectiveness of intravenous thrombolytic treatment in acute myocardial infarction. thrombolytic treatment
Lancet 1986; i:397-401.

16 Schiller NB, Shaha PM, Crawford M, et al. Recommendation for quantitation of the left ventricle by twodimensional echocardiography. If Am Soc Echocardiogr 1989;2:358-67.

17 Pace L, Perrone-Filardi P, Mainenti P, et al. Identification of viable myocardium in patients with chronic coronary artery disease using rest-redistribution thallium-201 tomography: optimal image analysis. f Nucl Med 1998;39:1869-74.
18 Rentrop PK, Cohen M, Blanke $\mathrm{H}$, et al. Changes in collateral filling immediately after controlled coronary artery occlusion by an angioplasty ball

19 Norusis MJ. SPSS for windows. Advanced statistics. Release 6.0. Chicago: SPSS Inc, 1993.

20 Sklenar J, Ismail S, Villanueva FS, et al. Dobutamine echocardiography for determining the extent of myocardial salvage after reperfusion. An experimental evaluation. Circulation 1994;90:1502-12

21 Elsasser A, Schlepper M, Klovekorn WP, et al. Hibernating myocardium. An incomplete adaptation to ischemia. Circulation 1997;96:2920-31.

22 Di Carli M, Sherman T, Khanna S, et al. Myocardial viability in asynergic regions subtended by occluded coronary arteries: relation to the status of collateral flow in patients with chronic coronary artery disease. $\mathcal{F} \mathrm{Am}$ Coll Cardiol 1994;23:860-8.

23 Sabia PJ, Powers ER, Ragosta $M$, et al. An association between collateral blood flow and myocardial viability in between collateral blood flow and myocardial viability in 1992;327:1825-31.

24 Cwaig JM, Cwaig M, Nagueh SF, et al. End-diastolic wall thickness as a predictor of recovery of function in myocardial hibernation: relation to rest-redistribution Tl-201 tomography and dobutamine stress echocardiography. $\mathcal{f}$ Am Coll Cardiol 35;5:1152-61.

25 Panza JA, Dislizian V, Laurienzo JM, et al. Relation between thallium uptake and contractile response to dobutamine. Implications regarding myocardial viability in patients with chronic coronary artery disease and left ventricular dysfunction. Circulation 1995;91:990-8.

\section{IMAGES IN CARDIOLOGY}

\section{An unusual hyperkalaemia induced block}

The tracing below was recorded from a patient with a DDD pacemaker and hyperkalaemia $(7.8 \mathrm{mEq} / 1)$ caused by acute renal failure. The atrial mechanism is sinus at a rate of $110 / \mathrm{min}$. Every sinus $\mathrm{P}$ wave is followed by a ventricular spike, but several of these fail to depolarise the myocardium. The paced QRS complexes are extremely wide, measuring about 0.38 seconds. There is a basic $2: 1$ block of artificial stimuli; following a ventricular capture, the ensuing spike falls at the apex of the $\mathrm{T}$ wave during the refractory period. This is caused by the extreme prolongation of the ventricular depolarisation and repolarisation process. In addition, spike-toQRS intervals show a gradual lengthening, ending in block of two consecutive stimuli.
The association of 2:1 block with progressive prolongation of the conduction intervals up to block of two or three consecutive impulses is known as "alternating Wenckeback periodicity". This phenomenon is well recognised in atrioventricular conduction, particularly in atrial flutter, but has not been hitherto observed in conduction between an artificial pacemaker and the ventricular myocardium.

Correction of the hyperkalaemia was followed by normal ventricular capture and narrowing of the QRS complexes.

FRANCESCO LUZZA SCIPIONE CARERJ GIUSEPPE ORETO oretogmp@tin.it
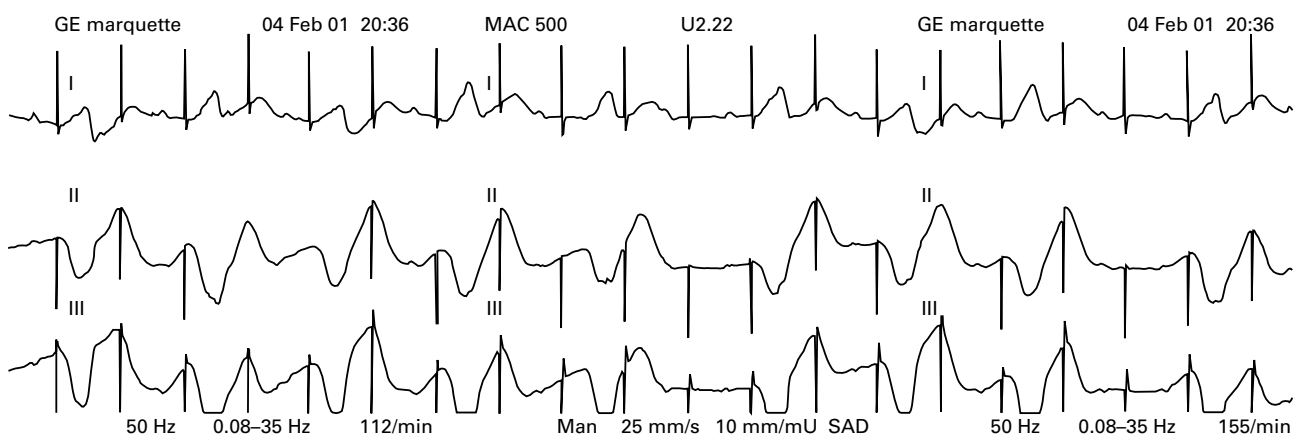